Tao Fu, Jin-Xia Ma, Qing-Lin Li, Ping Wei, Ji-Xing Zhao and Li Zhao*

\title{
Crystal structure of (E)-1-\{4-[(4-fluoro-2- hydroxybenzylidene)amino]phenyl\}ethanone $O$-methyl oxime, $\mathrm{C}_{16} \mathrm{H}_{15} \mathrm{FN}_{2} \mathrm{O}_{2}$
}

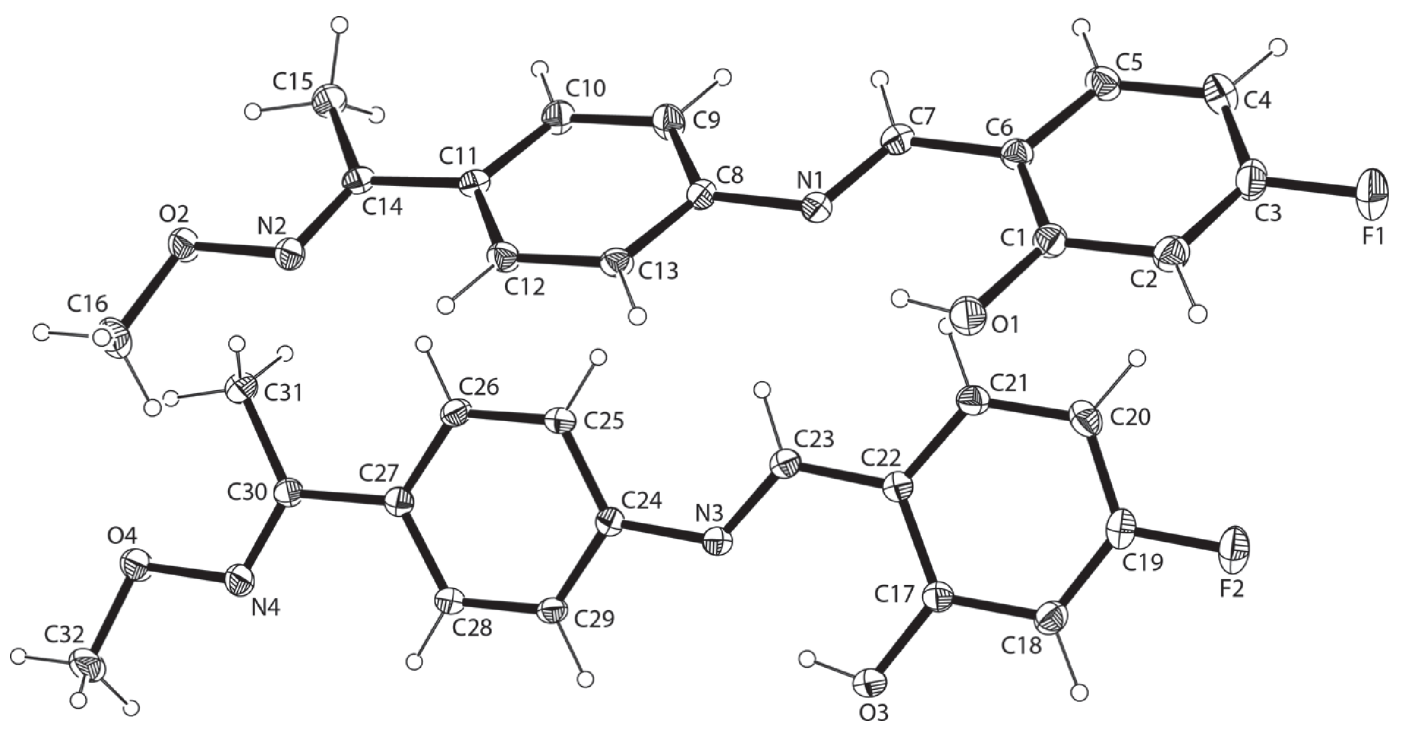

https://doi.org/10.1515/ncrs-2019-0614

Received August 25, 2019; accepted November 22, 2019; available online December 20, 2019

\section{Abstract}

$\mathrm{C}_{16} \mathrm{H}_{15} \mathrm{FN}_{2} \mathrm{O}_{2}$, monoclinic, $P 2{ }_{1} / c$ (no. 14), $a=6.0573(3) \AA$, $b=13.8341(6) \AA, \quad c=33.0876(15) \AA, \quad \beta=92.837(2)^{\circ}, \quad Z=8$, $V=2769.3(2) \AA^{3}, \quad R_{\mathrm{gt}}(F)=0.0581, \quad w R_{\text {ref }}\left(F^{2}\right)=0.1605$, $T=173(2) \mathrm{K}$.

\section{CCDC no.: 1967409}

The asymmetric unit of the title structure is shown in the figure. Table 1 contains crystallographic data and Table 2 contains the list of the atoms including atomic coordinates and displacement parameters.

*Corresponding author: Li Zhao, School of Chemical and Biological Engineering, Lanzhou Jiaotong University, Lanzhou 730070, P.R. China, e-mail: zhaoli_72@163.com. https://orcid.org/00000002-9898-9552

Tao Fu, Jin-Xia Ma, Qing-Lin Li and Ping Wei: Lanzhou Jiaotong University, School of Chemical and Biological Engineering, Lanzhou 730070, P.R. China

Ji-Xing Zhao: Shihezi University, Analysis and Testing Center, Xinjiang 832003, P.R. China

๑ Open Access. (๖ 2019 Tao Fu et al., published by De Gruyter. (c))BY License.
Table 1: Data collection and handling.

\begin{tabular}{ll}
\hline Crystal: & Yellow block \\
Size: & $0.19 \times 0.17 \times 0.14 \mathrm{~mm}$ \\
Wavelength: & Mo $K \alpha$ radiation $(0.71073 \AA)$ \\
$\mu:$ & $0.10 \mathrm{~mm}^{-1}$ \\
Diffractometer, scan mode: & Bruker D8 Venture, $\varphi$ and $\omega$ \\
$\theta_{\max }$, completeness: & $25.0^{\circ}, 99 \%$ \\
$N(h k l)_{\text {measured }}, N(h k l)_{\text {unique }}, R_{\text {int }}:$ & $19662,4869,0.063$ \\
Criterion for $I_{\text {obs }}, N\left(h k l_{\text {gt }}:\right.$ & $I_{\text {obs }}>2 \sigma\left(l_{\text {obs }}\right), 3089$ \\
$N(\text { param })_{\text {refined }}:$ & 385 \\
Programs: & Bruker [1], SHELX [2], Olex2 [3] \\
\hline
\end{tabular}

\section{Source of material}

All chemicals were used without further purification. 1(4-Aminophenyl)ethan-1-one $O$-methyl oxime was prepared by a method reported earlier [4, 5]. 4-Fluoro-2-hydroxybenzaldehyde (140.2 $\mathrm{mg}, 1 \mathrm{mmol})$ dissolved in $\mathrm{EtOH}(5 \mathrm{~mL})$ was added to 1-(4-aminophenyl)ethanone $O$-methyl oxime (164.5 mg, $1 \mathrm{mmol}$ ) dissolved in EtOH $(5 \mathrm{~mL})$. After being stirred for $2 \mathrm{~h}$ at room temperature, the filtrate was allowed to stand for 21 days in a quiet environment. Several block clear yellow crystals were obtained (yield 71.4\%, m.p. 374-382 K). Anal. calcd. for $\mathbf{C}_{16} \mathbf{H}_{15} \mathbf{F N}_{2} \mathbf{O}_{2}$ : C, 67.12\%; H, 5.28\%; N, 9.78\%; Found: C, 67.84\%; H, 5.62\%; N, 9.31\%. 
Table 2: Fractional atomic coordinates and isotropic or equivalent isotropic displacement parameters $\left(\AA^{2}\right)$.

\begin{tabular}{|c|c|c|c|c|}
\hline Atom & $x$ & $y$ & $z$ & $\boldsymbol{U}_{\text {iso }}{ }^{*} / \boldsymbol{U}_{\text {eq }}$ \\
\hline F1 & $-0.0087(3)$ & $0.12484(14)$ & $0.50935(5)$ & $0.0667(6)$ \\
\hline F2 & $-0.4769(3)$ & $-0.13346(13)$ & $0.43786(5)$ & $0.0600(5)$ \\
\hline 01 & $-0.2008(3)$ & $0.17686(15)$ & $0.37224(5)$ & $0.0473(5)$ \\
\hline $\mathrm{H} 1$ & -0.146962 & 0.172605 & 0.349388 & $0.071^{*}$ \\
\hline 02 & $0.1567(3)$ & $0.15842(13)$ & $0.08796(5)$ & $0.0407(5)$ \\
\hline 03 & $-0.6599(3)$ & $-0.15860(16)$ & $0.29901(5)$ & $0.0502(6)$ \\
\hline H3 & -0.600291 & -0.155096 & 0.276662 & $0.075^{\star}$ \\
\hline 04 & $-0.2765(3)$ & $-0.12463(18)$ & $0.01773(5)$ & $0.0592(6)$ \\
\hline N1 & $0.0895(3)$ & $0.13760(14)$ & $0.32026(6)$ & $0.0323(5)$ \\
\hline N2 & $0.1054(4)$ & $0.16241(15)$ & $0.12901(6)$ & $0.0352(5)$ \\
\hline N3 & $-0.3579(3)$ & $-0.12255(14)$ & $0.24933(6)$ & $0.0323(5)$ \\
\hline N4 & $-0.3284(4)$ & $-0.13147(19)$ & $0.05872(6)$ & $0.0473(6)$ \\
\hline $\mathrm{C} 1$ & $-0.0477(5)$ & $0.15035(18)$ & $0.40104(8)$ & $0.0366(6)$ \\
\hline $\mathrm{C} 2$ & $-0.1042(5)$ & $0.15280(19)$ & $0.44138(8)$ & $0.0417(7)$ \\
\hline $\mathrm{H} 2$ & -0.245997 & 0.174142 & 0.448583 & $0.050^{*}$ \\
\hline C3 & $0.0502(5)$ & $0.1237(2)$ & $0.47011(8)$ & $0.0455(7)$ \\
\hline $\mathrm{C} 4$ & $0.2601(5)$ & $0.0938(2)$ & $0.46232(8)$ & $0.0477(8)$ \\
\hline H4 & 0.362685 & 0.074359 & 0.483420 & $0.057^{*}$ \\
\hline $\mathrm{C} 5$ & $0.3146(5)$ & $0.0933(2)$ & $0.42240(8)$ & $0.0415(7)$ \\
\hline H5 & 0.459215 & 0.074089 & 0.415948 & 0.050 * \\
\hline C6 & $0.1640(4)$ & $0.12031(18)$ & $0.39108(7)$ & $0.0332(6)$ \\
\hline $\mathrm{C7}$ & $0.2281(4)$ & $0.11814(18)$ & $0.34963(7)$ & $0.0342(6)$ \\
\hline $\mathrm{H} 7$ & 0.375936 & 0.101974 & 0.344029 & $0.041^{*}$ \\
\hline $\mathrm{C} 8$ & $0.1481(4)$ & $0.13528(17)$ & $0.27930(7)$ & $0.0310(6)$ \\
\hline C9 & $0.3459(5)$ & $0.1001(2)$ & $0.26563(8)$ & $0.0404(7)$ \\
\hline H9 & 0.454564 & 0.074736 & 0.284417 & $0.048^{*}$ \\
\hline C10 & $0.3856(4)$ & $0.10185(19)$ & $0.22475(8)$ & $0.0387(7)$ \\
\hline $\mathrm{H} 10$ & 0.522422 & 0.078087 & 0.216015 & $0.046^{\star}$ \\
\hline C11 & $0.2305(4)$ & $0.13740(17)$ & $0.19622(7)$ & $0.0296(6)$ \\
\hline C12 & $0.0329(4)$ & $0.17298(19)$ & $0.21016(7)$ & $0.0349(6)$ \\
\hline H12 & -0.076051 & 0.198458 & 0.191448 & $0.042^{\star}$ \\
\hline C13 & $-0.0060(4)$ & $0.17155(18)$ & $0.25087(7)$ & $0.0330(6)$ \\
\hline H13 & -0.142079 & 0.196061 & 0.259666 & $0.040^{\star}$ \\
\hline C14 & $0.2703(4)$ & $0.13629(17)$ & $0.15238(7)$ & $0.0312(6)$ \\
\hline C15 & $0.4872(5)$ & $0.1034(2)$ & $0.13741(8)$ & $0.0423(7)$ \\
\hline H15A & 0.515089 & 0.036525 & 0.145935 & $0.063^{*}$ \\
\hline H15B & 0.605642 & 0.145117 & 0.148694 & $0.063^{*}$ \\
\hline $\mathrm{H} 15 \mathrm{C}$ & 0.482681 & 0.107172 & 0.107803 & $0.063^{*}$ \\
\hline C16 & $-0.0404(5)$ & $0.1772(2)$ & $0.06377(8)$ & $0.0526(8)$ \\
\hline $\mathrm{H} 16 \mathrm{~A}$ & -0.007535 & 0.175041 & 0.035078 & $0.079^{*}$ \\
\hline $\mathrm{H} 16 \mathrm{~B}$ & -0.097106 & 0.241395 & 0.070342 & 0.079 * \\
\hline H16C & -0.151713 & 0.128214 & 0.069283 & $0.079^{*}$ \\
\hline C17 & $-0.5103(4)$ & $-0.13684(19)$ & $0.32899(8)$ & $0.0353(6)$ \\
\hline C18 & $-0.5697(5)$ & $-0.1462(2)$ & $0.36885(8)$ & $0.0418(7)$ \\
\hline H18 & -0.713108 & -0.167975 & 0.374882 & $0.050^{*}$ \\
\hline C19 & $-0.4177(5)$ & $-0.1235(2)$ & $0.39901(8)$ & $0.0411(7)$ \\
\hline $\mathrm{C} 20$ & $-0.2077(5)$ & $-0.09074(19)$ & $0.39253(8)$ & $0.0432(7)$ \\
\hline $\mathrm{H} 2 \mathrm{O}$ & -0.107172 & -0.074325 & 0.414402 & $0.052^{\star}$ \\
\hline $\mathrm{C} 21$ & $-0.1499(5)$ & $-0.08285(19)$ & $0.35293(8)$ & $0.0381(7)$ \\
\hline $\mathrm{H} 21$ & -0.005342 & -0.061242 & 0.347588 & $0.046^{*}$ \\
\hline $\mathrm{C} 22$ & $-0.2958(4)$ & $-0.10540(17)$ & $0.32041(7)$ & $0.0310(6)$ \\
\hline $\mathrm{C} 23$ & $-0.2254(4)$ & $-0.10107(17)$ & $0.27955(7)$ & $0.0332(6)$ \\
\hline $\mathrm{H} 23$ & -0.078258 & -0.081972 & 0.274815 & $0.040^{*}$ \\
\hline C24 & $-0.2936(4)$ & $-0.12098(17)$ & $0.20884(7)$ & $0.0302(6)$ \\
\hline $\mathrm{C} 25$ & $-0.0934(4)$ & $-0.08463(19)$ & $0.19611(8)$ & $0.0373(6)$ \\
\hline
\end{tabular}

Table 2 (continued)

\begin{tabular}{lrrrr}
\hline Atom & $\boldsymbol{x}$ & $\boldsymbol{y}$ & $\boldsymbol{z}$ & $\boldsymbol{U}_{\text {iso }}{ }^{*} \boldsymbol{U}_{\text {eq }}$ \\
\hline H25 & 0.013033 & -0.059566 & 0.215398 & $0.045^{*}$ \\
C26 & $-0.0498(4)$ & $-0.08507(18)$ & $0.15538(7)$ & $0.0359(6)$ \\
H26 & 0.087835 & -0.060800 & 0.147215 & $0.043^{*}$ \\
C27 & $-0.2021(4)$ & $-0.12009(17)$ & $0.12618(7)$ & $0.0319(6)$ \\
C28 & $-0.4003(4)$ & $-0.15880(18)$ & $0.13945(7)$ & $0.0332(6)$ \\
H28 & -0.505579 & -0.185420 & 0.120327 & $0.040^{*}$ \\
C29 & $-0.4433(4)$ & $-0.15852(18)$ & $0.17973(7)$ & $0.0330(6)$ \\
H29 & -0.578928 & -0.184660 & 0.187973 & $0.040^{*}$ \\
C30 & $-0.1600(4)$ & $-0.11467(18)$ & $0.08251(7)$ & $0.0334(6)$ \\
C31 & $0.0639(5)$ & $-0.0896(2)$ & $0.06844(8)$ & $0.0448(7)$ \\
H31A & 0.061275 & -0.091949 & 0.038821 & $0.067^{*}$ \\
H31B & 0.172709 & -0.135981 & 0.079646 & $0.067^{*}$ \\
H31C & 0.104235 & -0.024292 & 0.077648 & $0.067^{*}$ \\
C32 & $-0.4733(6)$ & $-0.1422(3)$ & $-0.00665(9)$ & $0.0754(12)$ \\
H32A & -0.582915 & -0.092238 & -0.001380 & $0.113^{*}$ \\
H32B & -0.532949 & -0.205816 & -0.000027 & $0.113^{*}$ \\
H32C & -0.439331 & -0.140666 & -0.035294 & $0.113^{*}$ \\
\hline
\end{tabular}

\section{Experimental details}

Hydrogen atoms were placed in their geometrically idealized positions and constrained to ride on their parent atoms.

\section{Comment}

More and more attention is paid to the synthesis of mononuclear or multinuclear transition metal complexes with Schiff base ligands. Since the Schiff base ligand has excellent coordination ability with transition metal, it forms a stable transition metal complex [5-9]. So, we predict that the title compound is an excellent Schiff base ligand [10].

The current work reports the synthesis of title compound, which is built up by two $\mathrm{C}_{16} \mathrm{H}_{15} \mathrm{FN}_{2} \mathrm{O}_{2}$ molecules in the asymmetric unit. Compared with the structure already reported, all bond lengths of the title compound are in normal ranges [10-13]. There is one strong intramolecular hydrogen bond in each of the two crystallographically independent molecules (see the figure): $\quad 01-\mathrm{H} 1 \cdots \mathrm{N} 1 \quad(\mathrm{~d}(\mathrm{~N} 1 \cdots \mathrm{H} 1)=1.831 \AA$, $\mathrm{d}(\mathrm{O} 1-\mathrm{H} 1)=0.84 \AA$ and $\mathrm{d}(\mathrm{O} 1 \cdots \mathrm{N} 1)=2.578 \AA)$ and $03-$ $\mathrm{H} 3 \cdots \mathrm{N} 3 \quad(\mathrm{~d}(\mathrm{~N} 3 \cdots \mathrm{H} 3)=1.819 \AA 2, \mathrm{~d}(\mathrm{O} 3-\mathrm{H} 3)=0.84 \AA$ and $\mathrm{d}(\mathrm{O} 3 \cdots \mathrm{N} 3)=2.568 \AA$ ) .

\section{References}

1. Bruker. APEX2, SAINT and SADABS. Bruker AXS Inc., Madison, WI, USA (2012).

2. Sheldrick, G. M.: A short history of SHELX. Acta Crystallogr. A64 (2008) 112-122.

3. Dolomanov, O. V.; Bourhis, L. J.; Gildea, R. J.; Howard, J. A. K.; Puschmann, H.: OLEX2: a complete structure solution, refinement and analysis program. J. Appl. Crystallogr. 42 (2009) 339-341. 
4. Kaczmarek, M. T.; Kubicki, M.: The trimorphic structure of $N, N^{\prime}$ bis(5-methylsalicylidene)-4-methyl-1,3-phenylenediamine. Acta Crystallogr. B70 (2014) 792-800.

5. Dong, W. K.; Gong, S. S.; Tong, J. F.; Sun, Y. X.; Wu, J. C.: Syntheses and structure of two copper(II) complexes with salicyl mono-oxime ligands. Chin. J. Inorg. Chem. 26 (2010) 1868-1874.

6. Li, Q. L.; Li, P. P.; Ma, J. X.; Zhao, J. X.; Zhao, L.: Crystal structure of bis\{2-((E)-((4-((E)-1-(methoxyimino)ethyl)phenyl) imino)methyl)phenolato- $\mathrm{K}^{2} \mathrm{~N}, \mathrm{O}$ \}zinc(II), $\mathrm{C}_{32} \mathrm{H}_{30} \mathrm{~N}_{4} \mathrm{O}_{4} \mathrm{Zn}$. Z. Kristallogr. NCS 233 (2018) 637-639.

7. Sun, Y. X.; Zhao, Y. Y.; Li, C. Y.; Yu, B.; Guo, J. Q.; Li, J.: Supramolecular cobalt(II) and copper(II) complexes with Schiff base ligand: syntheses, characterizations and crystal structures. Chin. J. Inorg. Chem. 32 (2016) 913-920.

8. Sun, Y. X.; Li, C. Y.; Yang, C. J.; Zhao, Y. Y.; Guo, J. Q.; Yu, B.: Two $\mathrm{Cu}(\mathrm{II})$ complexes with Schiff base ligands: synthesis, crystal structure, spectroscopic properties, and substituent effect. Chin. J. Inorg. Chem. 32 (2016) 327-335.

9. Ma, J. X.; Li, Q. L.; Li, P. P.; Zhao, J. X.; Zhao, L.: Synthesis and crystal structure of bis $\{((E)-((4-((E)-1-(m e t h o x y i m i n o)$ ethyl)phenyl)imino)methyl)-2-naphtholato- ${ }^{2} N, O$ \}nickel(II), $\mathrm{C}_{40} \mathrm{H}_{34} \mathrm{~N}_{4} \mathrm{NiO}_{4}$. Z. Kristallogr. NCS 233 (2018) 789-791.

10. Zhao, L.; Dong, X. T.; Cheng, Q.; Zhao, J. X.; Wang, L.: Synthesis, crystal structure and spectral properties of a $2 D$ supramolecular copper(II) complex with 1-(4-\{[(E)-3-ethoxyl2-hydroxybenzylidene]amino\}phenyl) ethanone oxime. Synth. React. Inorg. Met.-Org. Nano-Met. Chem. 43 (2013) 1241-1246.

11. Sun, Y. X.; Lu, R. E.; Li, X. R.; Zhao, Y. Y.; Li, C. Y.: A Schiff base ligand containing oxime group and its $\mathrm{Cu}(\mathrm{II})$ complex: syntheses and supramolecular structures. Chin. J. Inorg. Chem. 31 (2015) 1055-1062.

12. Ma, J. X.; Li, Q. L.; Li, P. P.; Zhao, J. X.; Zhao, L.: Synthesis and crystal structure of $(E)-1-(4-(((E)-5$-chloro-2hydroxybenzylidene)amino)phenyl)ethan-1-one $O$-methyl oxime, $\mathrm{C}_{16} \mathrm{H}_{15} \mathrm{ClN}_{2} \mathrm{O}_{2}$. Z. Kristallogr. NCS 233 (2018) 463-464.

13. Zhao, L.; Wang, F.; An, Q. Q.; Zhao, J. X.: Crystal structure of (E)-1-(4-(( $(E)-3,5$-dichloro-2-hydroxybenzylidene)amino)phenyl) ethan-1-one $O$-ethyl oxime, $\mathrm{C}_{17} \mathrm{H}_{16} \mathrm{Cl}_{2} \mathrm{~N}_{2} \mathrm{O}_{2}$. Z. Kristallogr. NCS 231 (2016) 1045-1046. 